\title{
Phenotypic evolution under Fisher's Fundamental Theorem of Natural Selection
}

\author{
Eric L. Charnov
}

Department of Biology, University of Utah, Salt Lake City, UT 84112 U.S.A.

Lande's (1982) equations for phenotypic evolution are derived as a linearized version of Fisher's Fundamental Theorem of Natural Selection. In this derivation the genetic covariance matrix is not necessarily a fixed object and is likely to alter as directional selection proceeds. Under stabilizing or equilibrium selection, the mean phenotypes take on values identical to those which would be predicted by an "optimization of fitness in the face of tradeoffs" approach. It is argued that optimization is a more powerful way to understand equilibrium or stabilizing selection.

In this note I will derive equations for the evolution of correlated characters under natural selection with the assumption that the genetical dynamics satisfies Fisher's Fundamental Theorem of Natural Selection. The resulting equations, identical to those of Lande, will then be applied to the situation of stabilizing or equilibrium selection. The results will be compared to the output of a corresponding optimization approach to predicting the equilibrium phenotypes.

Consider the following familiar function

$$
1=\sum_{x=1}^{\infty} \frac{l_{x} \cdot b_{x}}{\lambda^{x}}
$$

where $l_{x}=$ probability of being alive at age $x, b_{x}=$ birth rate at age $x, \lambda=e^{m}=$ measure of Darwinian fitness (essentially the geometric rate of increase) for a genotype having the history $\left(l_{x}, b_{x}\right)$ under consideration.

For derivation of (1), in the continuous as well as discrete form, see Charlesworth (1980).

The most general theorem in population genetics is that the rate of change of mean fitness $(\bar{\lambda}, \bar{m})$ in a population is equal to the genic (= additive genetic) variance in fitness. Or, in two forms (Crow and Kimura, 1970):

$$
\begin{aligned}
\Delta \bar{\lambda} & =\frac{V(\lambda)}{\bar{\lambda}} \\
\Delta \bar{m} & =V(m) .
\end{aligned}
$$

These dynamical equations ensure that $\bar{\lambda}$ (or $\bar{m}$ ) will be non-decreasing with time (since the variance is non-negative) and that change will cease when $V(\lambda) \rightarrow 0$. The equations are, of course, known to population geneticists as R. A. Fisher's Fundamental Theorem of Natural Selection. Crow and Kimura (1970) discuss its approximate validity for a wide variety of genetic systems.

In this article I will ask how a set of phenotypic characters evolves if the underlying genetics satisfies these dynamical equations. In particular, I will show that the linearized version of Fisher's theorem leads to the same dynamical equations for phenotypes as Lande's (e.g., 1982) quantitative genetics approach to phenotypic evolution, at least for slow selection.

Consider first the function

$$
\lambda\left(Z_{1} \ldots, Z_{k}\right)
$$

where $\lambda$ is considered a random variable which is a function of several other random variables. The $Z_{i}$ are phenotypic variables (e.g., birth rates, height, bone structure). We expand $\lambda$ in Taylor series around the average $Z$ values $\left(\bar{Z}_{i}\right)$, retain the linear terms and then calculate the mean and variance of this linear approximation $\left(\lambda_{A}\right)$. To begin we have

$$
\lambda_{A} \approx \lambda\left(\bar{Z}_{1}, \ldots, \bar{Z}_{k}\right)+\sum_{i=1}^{k} \frac{\partial \lambda}{\partial Z_{i}}\left(Z_{i}-\bar{Z}_{i}\right)
$$


where the $\partial \lambda / \partial Z_{i}$ are evaluated at $\bar{Z}_{i}$. Note that the mean of $\lambda_{A}$ is simply

$$
\lambda\left(\bar{Z}_{1}, \ldots, \bar{Z}_{k}\right)=\bar{\lambda}_{A}(\approx \bar{\lambda}) .
$$

To calculate the variance of $\lambda_{A}$, we rewrite it as

$$
\begin{aligned}
\lambda_{A} \approx & \lambda\left(\bar{Z}_{1}, \ldots, \bar{Z}_{k}\right)-\sum_{i=1}^{k} \frac{\partial \lambda}{\partial Z_{i}} \bar{Z}_{i} \\
& +\sum_{i=1}^{k} \frac{\partial \lambda}{\partial Z_{i}} Z_{i} .
\end{aligned}
$$

The only random variables in (7) are the additive terms

$$
\sum_{i=1}^{k} \frac{\partial \lambda}{\partial Z_{i}} Z_{i}
$$

(and here only the $Z_{i}$ 's since the $\partial \lambda / \partial Z_{i}$ are constants, evaluated at the $\bar{Z}_{i}^{\prime}$ 's). Thus the $\operatorname{Var} \lambda_{A}(\approx \operatorname{Var} \lambda)$ reduces to the variance of a sum of random variables (the $Z_{i}$ 's). However, the world is a bit more complicated; we don't want $\operatorname{Var} \lambda$, we want $V(\lambda)$, the additive genetic variance. Let each $Z_{i}$ be made of two parts, two other random variables:

$Z_{\mathrm{ig}}=$ additive genetic part

$Z_{\mathrm{ie}}=$ the rest (environmental, dominance, etc.)

So that

$$
Z_{i}=Z_{\mathrm{ig}}+Z_{\mathrm{ic}},
$$

We shall make two assumptions here: first, that $\bar{Z}_{\mathrm{ie}}=0$, and second, that $\operatorname{Cov}\left(Z_{\mathrm{ig}}, Z_{\mathrm{ie}}\right)=0$ [no environment-genotype interaction, and environmental effects alter the variance but not the mean of the $Z_{i}$ 's]. With these assumptions, we can rewrite (8) as:

$$
\sum_{i=1}^{k} \frac{\partial \lambda}{\partial Z_{i}} Z_{\text {ig }}+\sum_{i=1}^{k} \frac{\partial \lambda}{\partial Z_{i}} Z_{\text {ie }}
$$

The first and second summations are uncorrelated random variables. The variance of the first summation represents the additive genetic variance of $\lambda_{A}$ (or the approximate additive genetic variance of $\lambda$ ); thus, we have:

$$
\begin{aligned}
V(\lambda) \approx & \sum_{i=1}^{k} V\left(Z_{i}\right)\left(\frac{\partial \lambda}{\partial Z_{i}}\right)^{2} \\
& +2 \sum_{i<j} \operatorname{Cov}\left(Z_{i}, Z_{j}\right)\left(\frac{\partial \lambda}{\partial Z_{i}}\right)\left(\frac{\partial \lambda}{\partial Z_{j}}\right)
\end{aligned}
$$

where $V\left(Z_{i}\right)$ and $\operatorname{Cov}\left(Z_{i}, Z_{j}\right)$ refer to the additive genetic variances and covariances. The derivatives are evaluated at the means of the $Z$ 's. Indeed, the functions $\lambda\left(Z_{1}, \ldots, Z_{k}\right)$ and $\lambda\left(\bar{Z}_{1}, \bar{Z}_{2}, \ldots, \bar{Z}_{k}\right)$ will be exactly the same form.
Equation (10) is of course the linearized version of the dynamical equation

$$
\vec{\lambda} \Delta \vec{\lambda}=V(\lambda) \quad \text { (or equation (2)) }
$$

To write an equation for the change $\left(\Delta \bar{Z}_{i}\right)$ in mean $Z_{i}$, note from equation 6 that $\bar{\lambda}_{A}=$ $\lambda\left(\bar{Z}_{1}, \ldots, \bar{Z}_{k}\right)$. If $\bar{\lambda}_{A}$ is changing slowly due to selection we may write

$$
\bar{\lambda}_{A}(T+1) \approx \bar{\lambda}_{A}(T)+\sum_{i \cdots 1}^{k} \frac{\partial \bar{\lambda}_{A}}{\partial \bar{Z}_{i}} \cdot \Delta \bar{Z}_{i}
$$

or

$$
\bar{\lambda}_{\Lambda}(T+1)-\bar{\lambda}_{A}(T)=\Delta \bar{\lambda}_{A}=\sum_{i=1}^{k} \frac{\partial \bar{\lambda}_{A}}{\partial \bar{Z}_{i}} \Delta \bar{Z}_{i} .
$$

Now, since $\Delta \bar{\lambda} \approx \Delta \bar{\lambda}_{A}=V(\lambda) / \bar{\lambda}$, it appears that

$$
\frac{V(\lambda)}{\bar{\lambda}} \approx \sum_{i=1}^{k} \frac{\partial \bar{\lambda}_{A}}{\partial \bar{Z}_{i}} \cdot \Delta \bar{Z}_{i}
$$

Inspection of equation (10), the linear approximation for $V(\lambda)$, shows that each $\partial \vec{\lambda} / \partial \bar{Z}_{i}$ term is multiplied by a $V\left(Z_{i}\right) \cdot \partial \bar{\lambda} / \partial \bar{Z}_{i}$ term and a sum of similar covariance terms. Each $Z_{i}, Z_{j}$ pair has two covariance terms. If we assign one covariance term to each $Z$ of the pair, the resulting summation for each $Z_{i}$ should (through equation (12)) be equal to $\bar{\lambda} . \Delta \bar{Z}_{i}$. We have for $i=1, \ldots, k$

$$
\begin{aligned}
\bar{\lambda} \cdot \Delta \bar{Z}_{i}= & V\left(Z_{i}\right) \frac{\partial \bar{\lambda}_{A}}{\partial \bar{Z}_{i}} \\
& +\sum_{\substack{i=1 \\
j \neq i}}^{k} \operatorname{Cov}\left(Z_{i}, Z_{j}\right) \frac{\partial \bar{\lambda}_{A}}{\partial \bar{Z}_{j}}
\end{aligned}
$$

or (in matrix form)

$$
\begin{gathered}
\left(\begin{array}{cc}
V\left(Z_{i}\right) & \operatorname{Cov}\left(Z_{1} Z_{2}\right) \cdots \\
\operatorname{Cov}\left(Z_{1} Z_{2}\right) & V\left(Z_{2}\right) \cdots \\
\vdots & \vdots
\end{array}\right)\left(\begin{array}{c}
\partial \bar{\lambda}_{A} / \partial \bar{Z}_{1} \\
\vdots \\
\partial \bar{\lambda}_{A} / \partial \bar{Z}_{k}
\end{array}\right) \\
=\left(\begin{array}{c}
\bar{\lambda} \cdot \Delta \bar{Z}_{1} \\
\vdots \\
\bar{\lambda} \cdot \Delta \bar{Z}_{k}
\end{array}\right)
\end{gathered}
$$

The end result (equations (13) or (14)) is the same form as derived by Lande (1982). I think it useful to show a derivation of them which begins with Fisher's Fundamental Theorem $(\bar{\lambda} \Delta \bar{\lambda}=V(\lambda))$ and recovers the variance-covariance formalism simply as the linearized version of $V(\lambda)$. Note that to do so also required zero covariance between the additive genetic effects and other sources (e.g., under the rubric of "environmental") of variation 
in the characters. Otherwise, the step from equation (9) to (10) could not ignore the second summation in equation (9). Lande (1982) makes essentially the same assumption. The linearized equations should apply in the neighborhood of equilibrium points, since there genetic variance is small and selection slow.

There is one important way in which this derivation differs from the Lande formalism; here there is no assumption that in equation (14) the genetic covariance matrix is constant through time. Indeed there is nothing fundamental about the values in the matrix at all as they simply represent linear relationships between the phenotypic variables $\left(Z_{i}\right)$ appropriate in a particular generation. If the covariance matrix (call it $\boldsymbol{V}$ ) and the vector of selection gradients $\left(\partial \bar{\lambda} / \partial \bar{Z}_{i}\right.$, call it $\left.\boldsymbol{\partial \lambda}\right)$ are allowed to change during a bout of directional selection, the most likely outcome is that phenotypic change will take place until an equilibrium is reached; here equation (14) will equal zero, or $\boldsymbol{V} \cdot \boldsymbol{\partial} \boldsymbol{\lambda}=0$. One may reasonably ask just what will determine the elements of the covariance matrix at the equilibrium? A reasonable guess, not necessarily complete, is the sorts of tradeoffs and/or constraints commonly studied by evolutionary ecologists (discussed in Charnov and Stephens, 1988; and Mangel and Clark, 1988). Of course, genetic covariances between characters may also result from functional relationships favored by natural selection, or from genetic linkage itself. The matrix $\boldsymbol{V}$ may well be a crazy patchwork quilt of tradeoffs, genetic linkage and functional relations between characters.

In the appendix, I show how evolution will proceed in a two-dimensional example where the phenotypic variables are subject to a tradeoff (fig. A1). Only at equilibrium $(\boldsymbol{V} \cdot \boldsymbol{\partial} \boldsymbol{\lambda}=0)$ are the phenotypes forced to lie on the tradeoff or constraint surface (see fig. A1); this generates a negative genetic covariance (the covariance may well be positive during directional selection, particularly if selection is moving the phenotypes from the inside outward towards the constraint boundary). The appendix also shows that the equilibrium under the genetical equations $(\boldsymbol{V} . \boldsymbol{\partial} \boldsymbol{\lambda}=0)$ is the same for the mean phenotypes $\left(\bar{Z}_{i}\right)$ as is obtained by the "standard optimization of fitness in the face of tradeoffs" approach as typically applied by evolutionary ecologists (Charnov and Stephens, 1988). As independently shown by B. Charlesworth (unpublished manuscript) for the arbitrary $\mathrm{N}$-dimensional case the structure of the covariance matrix and selection differentials as imposed by the tradeoffs will in equilibrium
$(\boldsymbol{V} . \boldsymbol{\partial} \boldsymbol{\lambda}=0)$ be the same as the solution to the corresponding optimization problem; the two methods for finding the equilibrium phenotypes will give identical answers. They differ mainly in that the covariance matrix is simply a linear representation of the true tradeoff relations. Since most thought by evolutionary ecologists related to tradeoffs results in non-linear tradeoffs or constraints (e.g., Charnov, 1986), I have elsewhere suggested (Charnov and Stephens, 1988) that the tradeoffs themselves are the fundamental objects of evolutionary interest, at least with respect to stabilizing or equilibrium selection. The covariance representation of tradeoffs or constraints simply discards too much which is of basic interest (see also Bell and Koufopanou, 1986, p. 123).

If most of the time species are subject to equilibrium (stabilizing) selection, then bouts of directional change in phenotypes can also be studied using an optimization approach. Two possibilities present themselves. In the first, directional selection results when a constraint surface alters; the population then moves to a new equilibrium. In the process the genetic covariance matrix will be reorganized to reflect the tradeoffs at the new equilibrium. The second possibility is that while no constraint surface is altered, there exists more than one local optimum. Non-linear tradeoffs seem most likely to generate this condition. As Sewall Wright often suggested, drift generated by small local population size may well allow a species to shift from one local optimum to another.

Acknowledgements For comments or an earlier version of these ideas, I thank James J. Bull, Sharon Emerson, J. M. Emlen, B. Charlesworth, M. Geber, J. Seger, J. Maynard Smith, S. Stearns and Jacob Koella. All the mistakes are my own.

\section{REFERENCES}

BELL, G. AND KOUFOPANOU, V. 1986. The cost of reproduction. Oxford Surveys in Evolutionary Biology, 3, 83-131.

CHARNOV, E. L. 1986. Life history evolution in a "recruitment population": why are adult mortality rates constant? Oikos, $47,129-134$.

CHARNOV, E. L. ANID STFPHENS, D. W. 1988. On evolution of host selection in solitary parasitoids. Amer. Natur. 132, $707-722$.

CHARlesworth, B. 1980. Evolution in Age Structured Populations. Cambridge, N.Y.

Crow, J. F. And kimura, M. 1970. An Introduction to Population Genetics Theory. Harper \& Row, N.Y.

LANDE, R. 1982. A quantitative genetic theory of life history evolution. Ecology, 63, 607-615.

MANGEL, M. AND CLARK, C. 1988. Dynamic Modeling in Behavioral Ecology. Princeton Univ. Press, Princeton, N.J. 


\section{APPENDIX: EVOLUTION IN TWO DIMENSIONS}

Suppose we examine evolution with two traits $\left(Z_{1} \& Z_{2}\right)$ and assume they are related to fitness by

$$
\lambda=a Z_{1}+b Z_{2} \quad(a, b>0)
$$

Suppose further that $Z_{1}$ and $Z_{2}$ are constrained to be on or within the tradeoff curve of fig. A1. The equilibrium will fall on the curve since $\lambda$ increases with increasing $Z_{1}$ and $Z_{2}$; denote $Z_{2}=f\left(Z_{1}\right)$ on the tradeoff curve. The evolutionary ecologist's optimization solution for the equilibrium is to find $Z_{1}$ and $Z_{2}$ so that $\lambda$ is maximized subject to the tradeoff. For this two-dimensional case we may simply rewrite $\lambda$ as

$$
\lambda=a Z_{1}+b \cdot f\left(Z_{1}\right) .
$$

Setting $\partial \lambda / \partial Z_{1}=0$ yields the condition $-a / b=$ $\partial f / \partial Z_{1}$. As shown in the figure, this is the intersec-

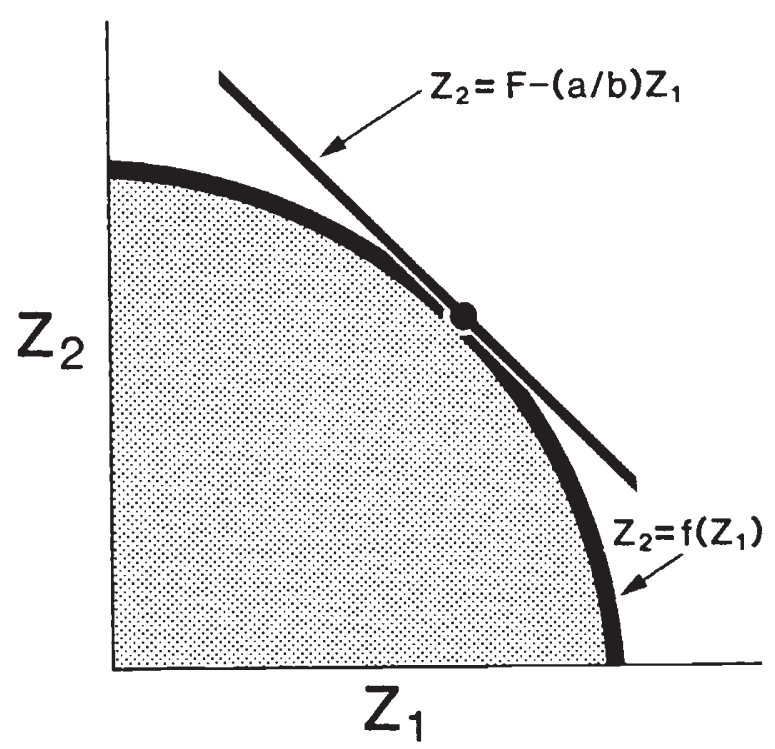

Figure A1 Phenotypic evolution in two dimensions. The possible phenotypes are in the stippled area, a tradeoff set. The equilibrium will be on the boundary of the tradeoff set, here called a tradeoff curve: $Z_{2}=f\left(Z_{1}\right)$. As shown in the text, the equilibrium will be the same under an optimization approach or a genetical dynamic approach $(\boldsymbol{V} \cdot \partial \boldsymbol{\lambda}=0)$. tion of a line with slope $-a / b$ with the tradeoff surface.

The dynamical version of the equilibrium is $\boldsymbol{V} . \boldsymbol{\partial} \boldsymbol{\lambda}=0$ or recalling that

$$
\boldsymbol{\partial \lambda}=\left(\begin{array}{c}
\frac{\partial \lambda}{\partial Z_{1}} \\
\frac{\partial \lambda}{\partial Z_{2}}
\end{array}\right),
$$

we have

$$
\left(\begin{array}{cc}
\operatorname{Var} Z_{1} & \operatorname{Cov} Z_{1} Z_{2} \\
\operatorname{Cov} Z_{1} Z_{2} & \operatorname{Var} Z_{2}
\end{array}\right)\left(\begin{array}{l}
a \\
b
\end{array}\right)=0
$$

In these equations, I have assumed for illustration that all the variance is additive genetic so that $V\left(Z_{i}\right)=\operatorname{Var}\left(Z_{i}\right)$. If these equations are to be satisfied at equilibrium, then the terms $\operatorname{Var} Z_{1}$, $\operatorname{Var} Z_{2}$, Cov $Z_{1} Z_{2}$ must assume particular formsimposed by the realization that the phenotypes are being pushed up against the constraint boundary $Z_{2}=f\left(Z_{1}\right)$. We may argue as follows (with reference to fig. A1). If the phenotypes occupy a small portion of the constraint boundary, $Z_{1}$ and $Z_{2}$ will be approximately linearly related or

$$
Z_{2}=M-\left(\partial f / \partial Z_{1}\right) . Z_{1} \text {. }
$$

Thus

$$
\operatorname{Var} Z_{2}=\left(\partial f / \partial Z_{1}\right)^{2} . \operatorname{Var} Z_{1} .
$$

With $Z_{2}=M-\partial f / \partial Z_{1} . Z_{1}$, the Corr. $\left(Z_{1} Z_{2}\right)=-1$. But

$$
\operatorname{Corr} .\left(Z_{1} Z_{2}\right)=\frac{\operatorname{Cov} Z_{1} Z_{2}}{\sqrt{\operatorname{Var} Z_{1}} \cdot \sqrt{\operatorname{Var} Z_{2}}}
$$

so that $\operatorname{Cov} Z_{1} Z_{2}=-\partial f / \partial Z_{1} . \operatorname{Var} Z_{1}$.

We have now written $\operatorname{Var} Z_{2}$ and $\operatorname{Cov} Z_{1} Z_{2}$ in terms of $\operatorname{Var} Z_{1}$ and the slope of the tradeoff curve. If we plug these values into equation $A 3$, they are appropriate to satisfy them equal to zero if and only if $\partial f / \partial Z_{1}=-a / b$, the same as we found under the optimization solution for the equilibrium. If fitness $(\lambda)$ is a non-linear function of $Z_{1}$ and $Z_{2}$, the same general methods apply, and the two answers are again the same. 